\title{
RELEVÂNCIA DO TREKKING E DO MOUNTAIN BIKE PARA O DESEMPENHO EM CORRIDA DE AVENTURA
}

\section{Relevance of trekking and mountain bike for the performance at adventure races}

\author{
Moacir Miorando Júnior, Flávio de Souza Castro, Marco Aurélio Vaz
}

\section{RESUMO}

O objetivo deste estudo foi comparar a relevância das modalidades de mountain bike e de trekking para o desempenho em prova de corrida de aventura. Seis atletas $(27,3 \pm 4,9$ anos; $73,0 \pm 5,5 \mathrm{~kg} ; 178,1 \pm 6,0 \mathrm{~cm} ; 22,9 \pm 0,9$ índice de massa corporal; $10,3 \% \pm 5,1 \%$ percentual de gordura corporal) realizaram testes de desempenho máximo nas modalidades de mountain bike e de trekking isoladamente, e, posteriormente, participaram de uma prova de corrida de aventura simulada. Correlações positivas e significativas foram encontradas entre o tempo de subida no teste de mountain bike com o tempo total de prova $(r=0,831 ; p<0,040)$, assim como com o tempo de prova na modalidade de mountain bike com o tempo total de prova $(r=0,833 ; p<0,039)$. Desta forma, foram observados indícios de que, entre as modalidades de mountain bike e de trekking, a mais relevante, em uma prova de corrida de aventura, seja o mountain bike.

Palavras-chave: Corrida de Aventura, Mountain Bike, Trekking.

\section{ABSTRACT}

The purpose of this study was to compare the relevance of the modalities of mountain bike and trekking at an adventure race. Six athletes $(27.3 \pm 4.9$ years; body mass: $73.0 \pm 5.5 \mathrm{~kg}$; height: $178.1 \pm 6.0 \mathrm{~cm}$; body mass index: $22.9 \pm 0.9$; body fat: $10.3 \% \pm 5.1 \%$ ) performed maximum tests on the modalities of mountain bike and trekking separately, and later they participated in a simulated adventure race. The positively correlated results were the ascent time in the mountain bike test with the total race time $(r=0.831 p<0.040)$ and the time of mountain bike modality in the race with the total race time $(r=0.833$ $p<0.039$ ). Thus, these results suggest that among mountain bike and trekking modalities, the most relevant modality at an adventure race is mountain bike.

Key words: Adventure Race, Mountain Bike, Trekking.

\section{INTRODUÇÃO}

A corrida de aventura é uma modalidade esportiva que envolve diversas atividades, tais como a orientação, o mountain bike, o trekking, as atividades aquáticas e as técnicas verticais. Este esporte se destaca dos demais pelo intenso contato com diferentes ambientes da natureza, sendo áreas de mata nativa, praias, montanhas e, inclusive, em regiões inóspitas, situações de extrema exigência psicológica, além de apresentar provas extremamente extenuantes, exigindo do atleta a manutenção de intensidade relativamente alta, durante longos períodos de tempo ${ }^{(1)}$. No Brasil, a corrida de aventura começou a ser praticada na última década, pois, com o grande êxodo rural, os esportes relacionados à natureza apresentaram, e continuam apresentando, grande procura.

A corrida de aventura obteve um grande desenvolvimento em um curto espaço de tempo, sendo que, atualmente, cada prova reúne, aproximadamente, duzentos atletas, agrupados em equipes. Atualmente, existe o Ranking Brasileiro de Corrida de Aventura (RBCA), entidade que tem o intuito de servir como parâmetro de qualidade entre as equipes brasileiras, esperando-se que, desta forma, as equipes se organizem e que seja criada uma federação ou associação em âmbito nacional.

Escola de Educação Física da Universidade Federal do Rio Grande do Sul - Porto Alegre - RS - Brasil.

Revista de Educação Física 2008 Set; 142:5-11. Rio de Janeiro (RJ) - Brasil.

Recebido em 11.04.2008. Aceito em 03.07.2008. 
TABELA 1

CARACTERÍSTICAS DA AMOSTRA.

\begin{tabular}{lccccc}
\hline Indivíduo & Idade & Massa $\mathbf{( k g )}$ & Estatura $\mathbf{( c m})$ & IMC & \% Gordura \\
\hline 1 & 26 & 70,2 & 176 & 22,6 & 10,2 \\
2 & 35 & 66,9 & 167 & 23,9 & 20,5 \\
3 & 24 & 71,8 & 182 & 21,6 & 8,0 \\
4 & 24 & 69,5 & 176 & 22,4 & 7,0 \\
5 & 23 & 80,0 & 182 & 24,1 & 7,8 \\
6 & 32 & 79,6 & 186 & 22,7 & 8,2 \\
Média & 27,3 & 73,0 & 178,1 & 22,9 & 10,3 \\
Desvio Padrão & 4,9 & 5,5 & 6,0 & 0,9 & 5,1 \\
\hline
\end{tabular}

O treinamento dos atletas desta modalidade permanece complexo e de difícil periodização, pois além de não existir um calendário elaborado com antecedência, são pouquíssimos estudos que podem auxiliar o trabalho dos profissionais que atuam na área. Os atletas e seus treinadores buscam fundamentação para elaborar seus treinos em outros esportes, principalmente no triathlon, modalidade que, devido às suas características de envolver três diferentes esportes, é, talvez, aquela que mais se aproxima da corrida de aventura.

O treinamento das modalidades isoladamente, para atletas deste esporte, visa o condicionamento aeróbio em alta intensidade, visto que as provas são extremamente longas e os atletas devem ter condições de manter, durante este período, uma alta intensidade. Sendo assim, utilizam diferentes vias metabólicas para o fornecimento de energia, sendo que, com a continuidade da atividade, a utilização de gordura aumenta gradativamente ${ }^{(2)}$.

As duas modalidades mais presentes na corrida de aventura, tendo um maior tempo de duração, são o trekking e o mountain bike. No entanto, pouco se sabe da contribuição relativa de cada uma dessas modalidades para o sucesso em uma prova de corrida de aventura. $\mathrm{O}$ conhecimento da modalidade mais relevante para o sucesso na prova pode proporcionar a realização de uma modulação de treino mais adequada.

A modalidade que se espera que apresente maior relevância para o desempenho em uma prova de corrida de aventura é a modalidade de mountain bike, já que é a modalidade em que o atleta atinge maior velocidade.

O objetivo deste estudo é comparar a relevância entre as modalidades de trekking e de mountain bike em uma prova de corrida de aventura.

\section{METODOLOGIA}

\section{Participantes}

Participaram do presente estudo, voluntariamente, seis atletas de corrida de aventura que treinavam regularmente há pelo menos um ano,apresentando nível intermediário e avançado de rendimento em observações durante provas do Circuito Gaúcho de Corrida de Aventura. Todos os participantes assinaram um termo de consentimento para estudos experimentais, onde constava toda metodologia do trabalho, seus riscos e benefícios, sendo este estudo realizado seguindo os preceitos e procedimentos éticos de pesquisa em seres humanos. Cada participante leu e assinou o termo de consentimento, após as explicações do objetivo, procedimentos experimentais, possíveis riscos e benefícios inerentes à pesquisa, conforme as diretrizes propostas na Resolução 196/96, do Conselho Nacional de Saúde, sobre pesquisas envolvendo seres humanos.

As características da amostra estão apresentadas na TABELA 1.

\section{Instrumentos}

$\mathrm{Na}$ avaliação física dos atletas, foram utilizadas: uma trena antropométrica WISO para mensurar a estatura; uma balança digital BF683W (Tanita) para verificar a massa corporal; e um plicômetro Cescorf Clínico para obtenção das dobras cutâneas dos participantes. Nos testes das modalidades de trekking e de mountain bike, assim como na prova simulada, foram utilizados: um cronômetro Cássio HS-3 para verificar o tempo dos indivíduos; e um GPS Garmin modelo eTrex Legend para verificação da altimetria e da distância percorrida.

\section{Métodos}

A coleta de dados foi dividida em três partes distintas, sendo que, inicialmente, foi realizada a verificação da 
massa corporal, da estatura e das dobras cutâneas dos participantes. Em seguida, foram realizados os testes de cada modalidade, individualmente, e, posteriormente, foi executada a prova de corrida de aventura simulada. Todos os atletas foram instruídos a não treinar exaustivamente nas 48 horas antecedentes, comparecendo alimentados e hidratados ao local dos testes. Os atletas foram, também, orientados a não consumirem estimulantes contendo cafeína antes dos testes. Todos os participantes utilizaram equipamento próprio durante toda coleta de dados.

Em um primeiro momento, foi realizada uma explanação, para os participantes, de como seria o desenrolar das atividades. A seguir, foi realizada a avaliação física dos indivíduos, estando os mesmos trajando somente calção, quando foi obtida a massa dos mesmos, bem como medida a estatura e as seguintes dobras cutâneas: tricipital, axilar, peitoral, subescapular, coxa, abdominal e supra-ilíaca. Para a definição do percentual de gordura corporal dos atletas, foi utilizado o protocolo de Guedes 7 dobras ${ }^{(3)}$.

Em um segundo momento, foram procedidos os testes das modalidades isoladamente, sendo que, primeiramente, foi realizado o teste de mountain bike. Neste teste, os indivíduos percorreram um trajeto de $3200 \mathrm{~m}$ (D), em uma estrada não pavimentada, sendo que os atletas partiram do ponto $A$, deslocaram-se até o ponto $B$ (parte de aclive) e retornaram até o ponto $A$ (parte de declive) para completar a distância. $O$ percurso apresentava um desnível de 180 metros de altitude $(\mathrm{H})$ entre o ponto $A$ e o ponto $B$. As altitudes e a distância foram medidas por meio de um GPS Garmin. O coeficiente de inclinação do percurso foi de $11,25 \%$ (H/Dx100). Todos os participantes utilizaram a relação das marchas e a calibragem dos pneus do modo a que estavam acostumados.

Após o teste de mountain bike, os indivíduos descansaram durante uma hora e, posteriormente, foram levados até o local do teste de trekking, sendo que este teste seguiu o mesmo protocolo do realizado na modalidade de mountain bike. A distância percorrida pelos atletas foi de $2800 \mathrm{~m}$, em uma trilha distinta, através do campo, sendo que os atletas saíram do ponto $A$, deslocaram-se até o ponto $B$ (parte de aclive) e retornaram até o ponto $\mathrm{A}$ (parte de declive) para completar tal distância. O percurso apresentava um desnível de 90 metros de altitude $(\mathrm{H})$ entre o ponto $A$ e o ponto $B$. As altitudes e a distância foram medidas através de um GPS Garmin. $O$ coeficiente de inclinação do percurso foi de $6,42 \%$ (H/Dx100).

Para finalizar a coleta de dados, após uma hora de intervalo, foi realizada a prova de corrida de aventura simulada. A prova foi considerada simulada devido ao fato do trajeto estar balizado com setas, indicando o caminho para que a modalidade de orientação não interferisse no resultado. Os atletas percorreram $17,15 \mathrm{~km}$ de trekking e 17,08 km de mountain bike. Durante a execução da prova, foram coletados os tempos dos participantes quando os mesmos passaram pela área de transição, local onde ocorreu a mudança de modalidade, sendo obtido o tempo total da prova. Nenhum atleta recebeu auxílio externo, estando somente autorizados a consumirem os alimentos que estavam carregando. Todos os testes ocorreram em um mesmo dia, das 9 hs às $17 \mathrm{hs}$, nas proximidades do Morro Ferra Braz, localizado no Município de Sapiranga, no Estado do Rio Grande do Sul.

\section{Análise de dados}

Os dados obtidos foram submetidos a procedimentos estatísticos, com cálculo de médias e desvios padrão, e verificação da normalidade dos dados (teste de Shapiro-Wilk). Logo em seguida, foi utilizado o teste de Correlação Linear Produto-Momento de Pearson, para $p<0,05$. O aplicativo SPSS v. 12.0 foi utilizado em todos os procedimentos.

\section{RESULTADOS}

A TABELA 2 apresenta os resultados dos testes das modalidades, nas fases de subida (A-B) e descida ( $B-A)$, tempo total no teste de cada modalidade, média de tempos e desvio padrão, sendo que os dados encontram-se em segundos.

Na TABELA 3, encontram-se os resultados da prova de cada indivíduo, sendo que os mesmos estão separados em modalidade e em tempo total de prova. Os dados encontram-se em segundos.

De todas as correlações testadas, foram significativas as entre o tempo total de prova e o tempo de subida, no teste de mountain bike, e entre tempo total de prova com o tempo da modalidade de mountain bike, durante a prova. Desta forma, a FIGURA 1 demonstra a correlação entre o tempo total de prova e o tempo de subida, no teste da modalidade de mountain bike de cada indivíduo, onde se pode observar uma correlação significativa. 
8 Revista de Educação Física 2008 Set; 142:5-11. Rio de Janeiro (RJ) - Brasil.

TABELA 2

RESULTADOS DOS TESTES DAS MODALIDADES ISOLADAS.

\begin{tabular}{lcccccc}
\hline & \multicolumn{3}{c}{ Mountain Bike } & \multicolumn{3}{c}{ Trekking } \\
\cline { 2 - 7 } ATLETA & A - B (s) & B - A (s) & Total (s) & A - B (s) & B - A (s) & Total (s) \\
\hline 1 & 614 & 170 & 784 & 437 & 398 & 835 \\
2 & 830 & 409 & 1239 & 525 & 419 & 944 \\
3 & 802 & 168 & 970 & 483 & 351 & 834 \\
4 & 707 & 127 & 834 & 468 & 371 & 839 \\
5 & 843 & 205 & 1048 & 446 & 343 & 789 \\
6 & 914 & 162 & 1076 & 508 & 402 & 910 \\
Média & 785 & 207 & 992 & 478 & 381 & 859 \\
Desvio Padrão & 107 & 102 & 159 & 41 & 29 & 57 \\
\hline
\end{tabular}

TABELA 3

RESULTADOS DA PROVA.

\begin{tabular}{lccccc}
\hline Modalidade de Mountain Bike & \multicolumn{2}{c}{ Modalidade de Trekking } & \multicolumn{2}{c}{ Tempo Total de Prova } \\
\hline Atleta & Tempo (s) & Atleta & Tempo (s) & Atleta & Tempo (s) \\
\hline 1 & 4110 & 1 & 6597 & 1 & 10707 \\
2 & 4945 & 2 & 6385 & 2 & 11330 \\
3 & 4610 & 3 & 6782 & 3 & 11392 \\
4 & 4020 & 4 & 6135 & 4 & 10155 \\
5 & 5220 & 5 & 6067 & 5 & 11287 \\
6 & 5124 & 6 & 7059 & 6 & 12183 \\
\hline
\end{tabular}

FIGURA 1

CORRELAÇÃO ENTRE O TEMPO TOTAL DE PROVA E O TEMPO DE SUBIDA NO TESTE DA MODALIDADE DE MOUNTAIN BIKE.

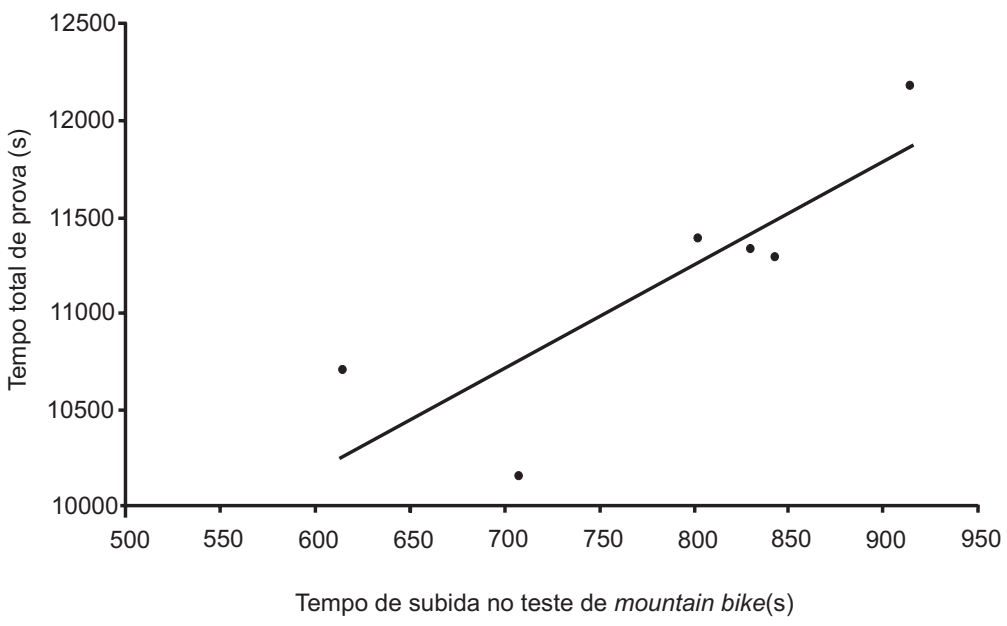

$r=0,831 p=0,040$. 
FIGURA 2

CORRELAÇÃO ENTRE O TEMPO TOTAL DE PROVA E O TEMPO DE PROVA DA MODALIDADE DE
MOUNTAIN BIKE.

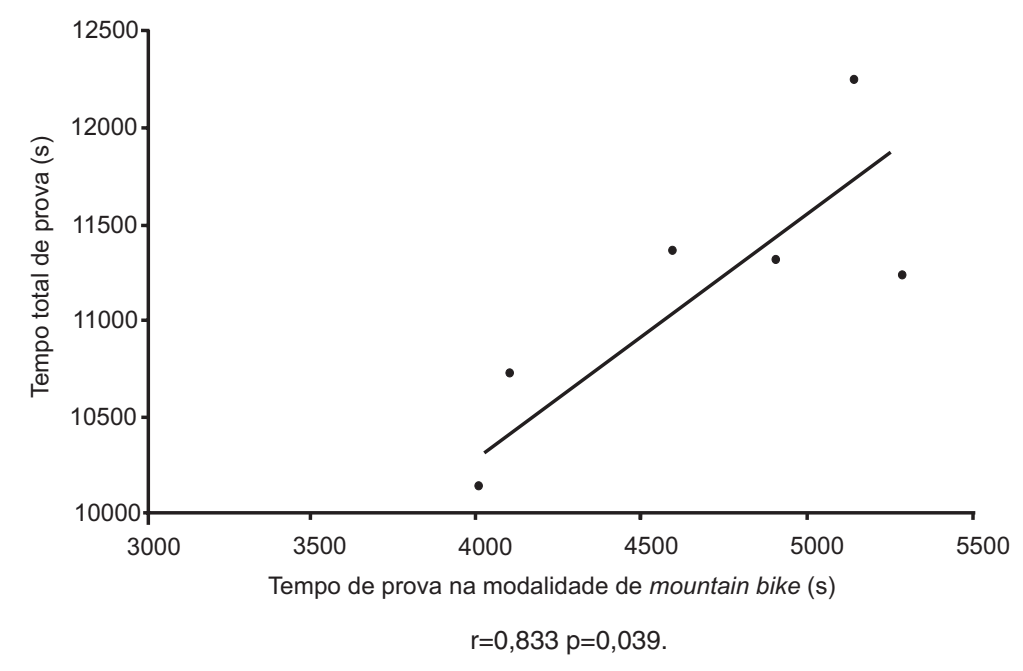

FIGURA 3

CORRELAÇÃO ENTRE O TEMPO TOTAL DE PROVA E O TEMPO DE SUBIDA NO TESTE DA MODALIDADE DE TREKKING.

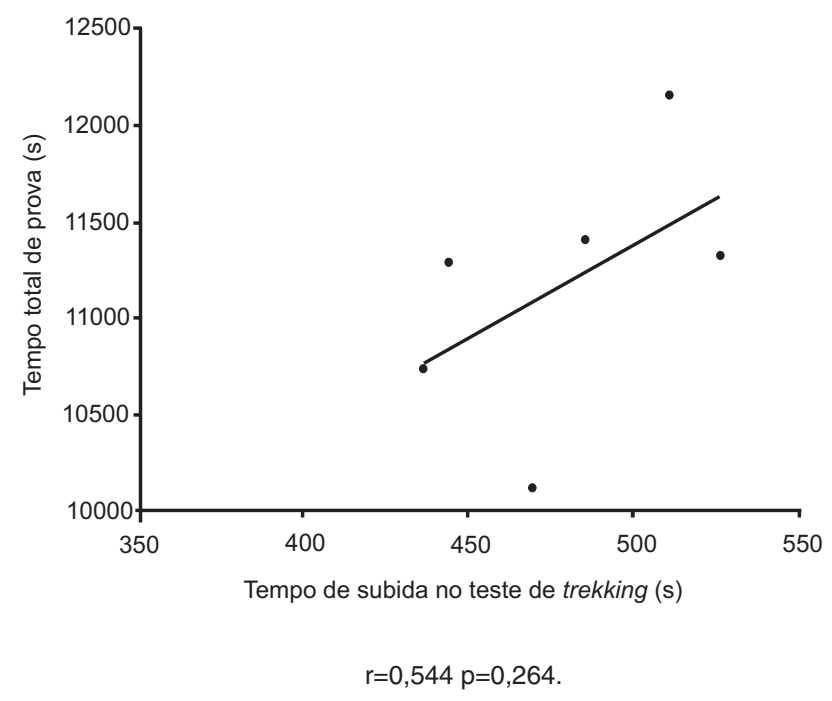

Na FIGURA 2, é observada a correlação do tempo total de prova com o tempo da modalidade de mountain bike durante a prova. Nota-se que os resultados, muito similares aos apresentados na FIGURA1, também demonstram uma correlação significativa. Desta maneira, torna-se evidente a importância da modalidade de mountain bike em uma prova de corrida de aventura.

A FIGURA 3 apresenta a correlação entre o tempo total de prova e o tempo de subida, no teste da modalidade de trekking. Percebe-se que esta correlação não é tão relevante quanto as apresentadas anteriormente.

Na FIGURA 4, está apresentada a correlação do tempo total de prova com o tempo da modalidade de trekking durante a prova. Esta correlação é mais relevante do que a apresentada na FIGURA 3, porém de menor importância quando comparada às apresentadas nas duas primeiras figuras, onde está envolvida a modalidade de mountain bike. 
FIGURA 4 CORRELAÇÃO ENTRE O TEMPO TOTAL DE PROVA E O TEMPO DE PROVA DA MODALIDADE DE TREKKING.

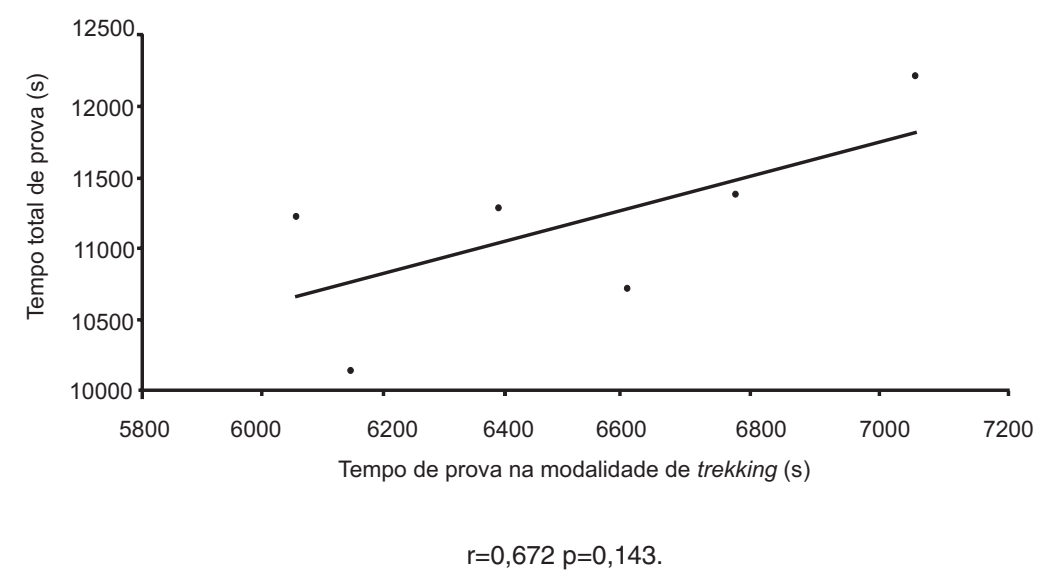

\section{DISCUSSÃO}

Sabe-se que o treinamento é um dos fatores determinantes no desempenho do atleta e que a especificidade deste treinamento é importante para um bom resultado, devido à melhora da potência aeróbia máxima e da economia de movimento ${ }^{(4)}$. Este estudo foi desenvolvido visando obter indícios de qual modalidade seria mais relevante para uma prova de corrida de aventura, pois, desta forma, os atletas, que necessitam treinar diversas modalidades, assim como seus técnicos, podem realizar um planejamento de treinamento mais específico.

Todos os atletas envolvidos no estudo treinavam há pelos menos um ano, e, desta maneira, apresentavam boa técnica de corrida e ciclismo, conforme observação realizada em provas do Circuito Gaúcho de Corrida de Aventura. No ciclismo, as técnicas para a estabilização do centro de massa, além das estratégias de mudanças de marchas, parecem ser fundamentais para o desempenho ${ }^{(5)}$. Além dos fatores supracitados, é de suma importância que sejam considerados fatores ambientais e o equipamento utilizado por cada atleta, pois tais elementos influenciam no desempenho das modalidades praticadas $^{(6)}$.

Os dados obtidos na avaliação antropométrica da amostra não apresentaram relação com o desempenho dos atletas, nas modalidades, isoladamente ou na prova, diferentemente do que Lee, Martin, Anson, Grundy e Hahn ${ }^{(7)}$ encontraram em seu estudo com mountain bikers.

Nos resultados obtidos nos testes de campo, pode-se observar que as correlações do tempo de subida no teste de mountain bike e o tempo de prova do mountain bike, com o tempo total de prova, respectivamente 0,831 e 0,833, são muito superiores às correlações do tempo de subida no teste de trekking e o tempo de prova da modalidade de trekking, com o tempo de prova, respectivamente 0,544 e 0,672 . Desta forma, os resultados apresentam indícios que ratificam a hipótese do presente estudo, onde é apontada a modalidade de mountain bike, em comparação com o trekking, como sendo a de maior relevância em uma prova de corrida de aventura em que a quilometragem das duas modalidades seja semelhante. Não existe pesquisa semelhante com o desporto de corrida de aventura, porém, o estudo de Dengel, Flynn, Costill e Kirwan ${ }^{(8)}$ apontou indícios de que a corrida é a modalidade determinante no triathlon.

Conforme observação durante provas do Circuito Gaúcho de Corrida de Aventura, a modalidade de mountain bike, quando comparada à modalidade de trekking, apresenta uma diferença de velocidade média de deslocamento superior entre um atleta bem condicionado e outro com um condicionamento inferior, mesmo possuindo um tempo de prova menor para uma mesma quilometragem. Em algumas oportunidades, os atletas não tão bem preparados não possuem condições de subir trechos pedalando, necessitando descer da bicicleta e empurrá-la. Assim, a bicicleta torna-se um empecilho ao invés de um auxílio, diminuindo, ainda mais, a velocidade de deslocamento.

Outro ponto que merece discussão é que durante a modalidade de mountain bike, normalmente, os deslocamentos são realizados em estradas não pavimentadas, facilitando, desta forma, a modalidade de 
orientação. Entretanto, a modalidade de orientação é mais exigida durante o trekking, pois grande parte do percurso é realizada através campo, onde nem sempre existem trilhas definidas para os atletas utilizarem. Desta maneira, a velocidade de deslocamento durante a modalidade de trekking acaba sendo diminuída, em alguns momentos, por fatores externos, tais como a dificuldade na modalidade de orientação e em terrenos de difícil progressão.

A revisão de literatura deste estudo possibilitou verificar que a relevância das modalidades em corrida de aventura é uma área pouco pesquisada, apresentando diversos fatores ainda não abordados e desconhecidos para as comunidades desportivas e de ciências do esporte. Por esta razão, não foi possível uma comparação de metodologia e, até mesmo, de resultados para ratificar os indícios do presente trabalho.

\section{CONCLUSÃO}

Os resultados deste estudo sugerem que a modalidade de mountain bike possui maior relevância em uma prova de corrida de aventura, quando comparada à modalidade de trekking, devido à maior diferença na velocidade de deslocamento entre os atletas. Sendo assim, na periodização de treinamento de atletas de corrida de aventura, deve ser priorizada a modalidade de mountain bike.

Devido à escassez de estudos nesta área, permanecem questões quanto à relevância da quilometragem de cada modalidade, sexo e nível de treinamento dos atletas, assim como a influência da presença das demais modalidades, pertencentes à corrida de aventura, no desempenho em prova simulada.

\section{REFERÊNCIAS BIBLIOGRÁFICAS}

1. Ferreira DM, Andrade A, Portela A. Caracterização do perfil sócio-econômico, motivacional, stress e ansiedade percebidos de competidores de corridas de aventura. EFDEPORTES [revista digital] 2005; 91. Disponível em: URL: http://www.efdeportes.com/efd91/aventura.htm(12 jun. 2007).

2. Rogatto GP. Perfil metabólico durante o exercício físico: influência da intensidade e da duração do esforço sobre a utilização de substratos energéticos. Revista digital EFDEPORTES 2002; 54. Disponível em: URL: http:// www.efdeportes.com/efd54/metab.htm(20 jul. 2007).

3. Guedes DP. Composição corporal: princípios, técnicas e aplicações. Florianópolis: CEITEC; 1992.

4. Caputo F, Stella SG, Mello MT, Denadai BS. Índices de potência e capacidade aeróbia obtidos em cicloergômetro e esteira rolante: comparações entre corredores, ciclistas, triatletas e sedentários. Revista Brasileira de Medicina do Esporte 2003; 9(4):223-30.

5. Machado CEP, Caputo F, Lucas RD, Denadai BS. Fatores fisiológicos e antropométricos associados com a performance em subida no ciclismo off-road. Revista Brasileira Ciência e Movimento 2002; 10(4):35-40.

6. Dias MR, Lima JRP, Novaes JS. Cadência de pedalada no ciclismo: uma revisão de literatura. Motricidade 2007; 3(1):270-8.

7. Lee H, Martin DT, Anson JM, Grundy D, Hahn AG. Physiological characteristics of successful mountain bikers and professional road cyclists. J Sports Sci 2002; 20:1001-8.

8. Dengel DR, Flynn MG, Costill DL, Kirwan JP. Determinants of success during triathlon competition. Research Quarterly for Exercise and Sport 1989; 60(3):234-8.

\section{Endereço para correspondência:}

Avenida Nilópolis 210 / 503 - Petrópolis

Porto Alegre - RS - Brasil

CEP 90460-050

Tel.: (51) $33317221 / 93347149$

e-mail: netos.13@ hotmail.com souza.castro@ufrgs.br marcovaz@esef.ufrgs.br 